\title{
Microcolumn liquid chromatography coupled with solid phase micro extraction (SPME/micro-LC) for the analysis of benzodiazepines in human urine
}

\author{
K. Jinno ${ }^{1}$, M. Taniguchi ${ }^{1}$, H. Sawada ${ }^{1}$ and M. Hayashida ${ }^{2}$ \\ ${ }^{1}$ School of Materials Science, Toyohashi University of Technology, Toyohashi 441-8580, Japan \\ ${ }^{2}$ Department of Legal Medicine, Nippon Medical School, Tokyo 113-0022, Japan
}

\begin{abstract}
The interface technique between SPME and micro-LC has been evaluated for benzodiazepines analysis in human urine. Three SPME fiber coatings such as polyacrylate (PA), carbowax/template (CW/TEP) and sol-gel $\mathrm{C}_{11}$ polydimethylsiloxane (PDMS) were compared in the performance for SPME process such as extraction efficiency, extraction time and total analysis time required. As the results using the CW/TEP fiber for SPME and $1.0 \mathrm{~mm}$ i.d. LC column as the separation medium the analysis of benzodiazepines in patient's urine can be performed in 2 hours with very low solvent consumption less than $1.5 \mathrm{~mL}$ solvents in whole analytical procedures.
\end{abstract}

creening and confirmation of drugs are important to clarify their therapeutic and toxic effects. Benzodiazepines are usually used as tranquilizers of the central nervous system in the clinical treatment, and, in the same time they will produce side or toxic effects in the case of overdose. Thus, the toxicological drug confirmation is demanded for these drugs to provide valuable information to physicians who are faced with drug poisoning patients [1].

In human fluids analysis a sample preparation technique is often necessary to extract and concentrate organic compounds of interest from the biological matrix. One of the technique for this purpose is solid phase micro extraction (SPME), as solvent free sample preparation technique, which has been recently developed [2]. In SPME, the outer surface of solid fused-silica fiber was coated with a selective polymeric material. The extraction operation is carried out by simply dipping the coated fiber into sample matrix and allowing time for the partition equilibrium to be established. The amounts of an analyte extracted by the coating could be described by Nernst's partition law. The sensitivity of the method is mostly governed by partition coefficient of an analyte between the coating and the sample matrix. Selective extraction can be achieved by using appropriate type of polymeric materials that exhibit high affinity toward the target analytes. Therefore the choice of coating is important for the method development in SPME. SPME is normally followed by gas chromatography (GC), in which the extracted analytes are thermally desorbed in the GC injector port for introduction onto a GC column [3-7]. However, GC analysis is not suitable for non-volatile or thermally unstable compounds such as benzodiazepines. Therefore in order to apply SPME to the compounds, it is needed that SPME should couple with other separation methods such as liquid chromatography (LC) or capillary electrophoresis (CE). Recently SPME coupled with LC and CE has been reported [8-12]. In our previous study SPME/semi-microcolumn LC (1.5 mm i.d.) was evaluated for drug analysis and the work showed that the method could analyze benzodiazepines in human urine without tedious and complex analytical procedures [13]. In addition, the consumption of hazardous organic solvents was reduced through the analytical process because semi-microcolumn LC needs less solvent as mobile phase than conventional LC in addition to the fact that SPME is a solvent free sample preparation technique.

In this paper, much smaller diameter LC columns such as $1.0 \mathrm{~mm}$ i.d. were used to be coupled with SPME (SPME/micro-LC) to much reduce the consumption of organic solvents for the benzodiazepines analysis. This SPME/micro-LC system becomes very important analytical method for this kind of toxic drug analysis in terms of speed, less laborious and ecological means.

\section{Experimental}

\section{Materials and reagents}

The holder and the assembly of SPME device for manual sampling were purchased from Supelco (Bellefonte, PA, USA). The $1.0 \mathrm{~cm}$ long fibers used were coated with $85 \mu \mathrm{m}$ thickness polyacrylate (PA) and $50 \mu \mathrm{m}$ thickness carbowax/template (CW/TEP) (Supelco). A new type sol-gel $\mathrm{C}_{11}$ PDMS with $50 \mu \mathrm{m}$ thickness was gifted from Supelco. This sol-gel type fiber has $C_{11}$ functional group. The new fibers were conditioned by immersing them into acetonitrile until interfering peaks were disappeared in LC chromatograms.

All solvents were reagent grade purchased from Kishida Chemical (Osaka, Japan) and deionized water obtained from a Milli-Q water system (Millipore, Tokyo, Japan).

\section{Apparatus}

Micro-LC was performed with an Nanospace SI-1 (Shiseido, Tokyo, Japan), which consists of a pump, a UV-Vis detector, a column oven and a degasser. Capcell pak ODS columns $(250 \mathrm{~mm} \times 1.0 \mathrm{~mm}$ i.d., and $150 \mathrm{~mm} \times 1.0 \mathrm{~mm}$ i.d.) connected with Rheodyne 7125 injector (Cotati, CA, USA) with a $1 \mu \mathrm{L}$ loop were used. The flow rate of the 
mobile phase was $50 \mu \mathrm{L} / \mathrm{min}$ and the column temperature was controlled at $35^{\circ} \mathrm{C}$. Mobile phase was acetonitrilebuffer (5 mM phosphate buffer) (40-60) and the detection wavelength was at $220 \mathrm{~nm}$. BORWIN chromatography software (Jasco, Tokyo, Japan) was used for data acquisition and handling.

\section{SPME /micro-LC procedure}

A $15 \mathrm{~mL}$ Milli-Q water spiked with standard drugs was placed in a $20 \mathrm{~mL}$ vial together with the cylindrical-shaped stirrer bar $(4 \times 6 \mathrm{~mm})$ and $6 \mathrm{~g}$ sodium chloride, and sealed. The fiber was introduced carefully into the aqueous phase and stirred at $1200 \mathrm{rpm}$ at $60^{\circ} \mathrm{C}$ for 1.0 hour. Then the fiber was withdrawn and introduced for a period of $30 \mathrm{~min}$ into the specially designed interface developed in our laboratory filled with the mobile phase as the desorption solvent for LC analysis. The details of procedure were described in our previous publication [13].

\section{Results and discussion}

The choice of an appropriate fiber coating is essential for the SPME method development. Depending on the molecular weight and polarity of the analytes to be extracted, the sensitivity of each fiber coating is different. Three types of fiber coatings such as the sol-gel $\mathrm{C}_{11}$ poly(dimethylsiloxane) (PDMS), the polyacrylate (PA), and the carbowax/template (CW/TEP), were evaluated for benzodiazepines analysis using SPME/micro-LC in this work.

The established conditions of SPME to investigate the performance of three fiber coatings are as follows: extraction temperature is $60{ }^{\circ} \mathrm{C}$, extraction time is $60 \mathrm{~min}$, the matrix is conditioned on phosphate buffer $(\mathrm{pH}$ 6.8) and saturated, desorption time is $30 \mathrm{~min}$, desorption solvent is the mobile phase and its flush volume is $6 \mu \mathrm{L}$. Most of these parameters were optimized in our previous work [13]. Saturation with salt can be used to not only improve the extraction efficiency, but also to normalize random salt concentration in human fluids. The 1,4-benzodiazepines such as triazolam, flunitrazepam and diazepam are selected as the probe and these compounds are considered as weak bases. Although they have certain degree of hydrophobicity, they can form ionic species easily, and thus can be classified as ionogenes [14]. Therefore the adjustment of matrix $\mathrm{pH}$ to that neutral forms of benzodiazepines are formed is significant to extract effectively this group of compounds. The extraction performance of three fibers for three compounds were evaluated under the optimum SPME condition and the results are summarized in figure 1 . As can be seen in figure 1 , the sol-gel $\mathrm{C}_{11}$ PDMS fiber gives the highest extraction efficiency in the three coatings for benzodiazepines. It is the well known fact that the sol-gel type coating enhances the surface area and very strong thermal stability relative to commercial PDMS fiber coating. The presence of hydroxyl group in the sol-gel structure makes the coating more polar relative to conventional PDMS coating. Therefore the sol-gel type coating can extract both polar and non-polar compounds [15]. Although the more polar CW/TEP fiber coating was successful in extracting benzodiazepines, the amounts of the analytes extracted by the CW/TEP is lower than the sol-gel $\mathrm{C}_{11}$ PDMS fiber coating by this evaluation.
The CW/TEP fiber coating, however, showed are higher extraction efficiency than PA fiber which was used to extract benzodiazepines in our previous study [13]. The CW/TEP is more polar than PA and has been used to extract alkylphenol ethoxylate surfactant [16]. It has been concluded by these results that the sol-gel $\mathrm{C}_{11}$ PDMS and the CW/TEP fibers are much better coatings than PA for benzodiazepines analysis.

The absorption-time profile of the sol-gel $\mathrm{C}_{11}$ PDMS and the CW/TEP fibers were then studied by monitoring the LC peak area counts as a function of extraction time shown in figure 2. It takes $120 \mathrm{~min}$ to reach the equilibrium when the sol-gel $\mathrm{C}_{11}$ PDMS fiber was used, while the CW/TEP requires $60 \mathrm{~min}$ to reach the equilibrium except the case of diazepam. It is considered that the extraction speed is controlled by mass transfer from the sample matrix to the fiber coating in SPME, and also the mass transfer rate can be determined by means of the diffusion of analytes in the coatings if the sample matrix is perfectly agitated. Therefore the fiber coating thickness influences the time required to the equilibrium and the amount of analytes to be extracted [17]. The thickness of these two fibers is the same as $50 \mu \mathrm{m}$, however. Therefore it seems that the difference of the time to the equilibrium is caused by the difference of characteristics of the coatings.

The carryover which is the ratio of the amount of analytes remaining on the fiber after the first desorption to the amount of the total analytes absorbed was also investigated. After first 30 min desorption was done, the carryover of triazolam, flunitrazepam and diazepam with CW/TEP fiber was $9.1,14.4$ and $18.6 \%$, respectively. For the sol-gel $\mathrm{C}_{11}$ PDMS fiber the carryover was 20.3, 9.9, and 13.3, respectively. Although the carryover is reduced by the longer desorption time, the desorption time longer than $30 \mathrm{~min}$ is too long for the actual analysis. Recently Janusz et al. [18] reported that heating during the desorption process in SPME/LC combination was very effective to reduce the carryover on the fiber coating. This fact means that increasing

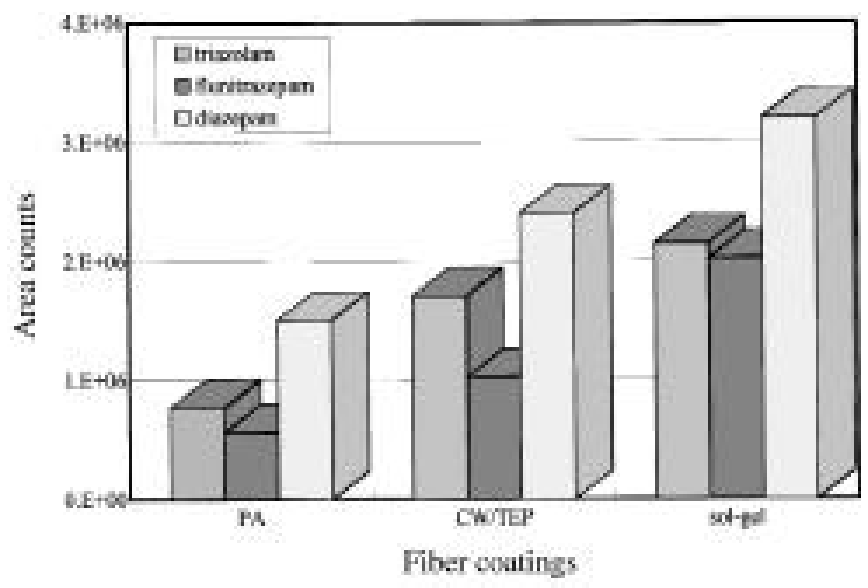

Figure 1. Extracted amounts indicated as the peak area counts in LC chromatogram by using different SPME fiber coatings. Concentration of each analyte is $0.1 \mu \mathrm{g} / \mathrm{mL}$. Extraction time; $60 \mathrm{~min}$, extraction temperature; $60{ }^{\circ} \mathrm{C}$, desorption time; $30 \mathrm{~min}$, desorption solvent; mobile phase (acetonitrile/5 $\mathrm{mM}$ phosphate buffer $=40 / 60$ ). 
the temperature in desorption process can reduce the distribution constant between the fiber coating and the desorption solvent and increase the diffusion rate in the process.

Based on such literature information one can consider that the reduction of carryover amount can be realized by using preheated solvent for the desorption process. In this work the hot mobile phase buffer solution heated at $60{ }^{\circ} \mathrm{C}$ was
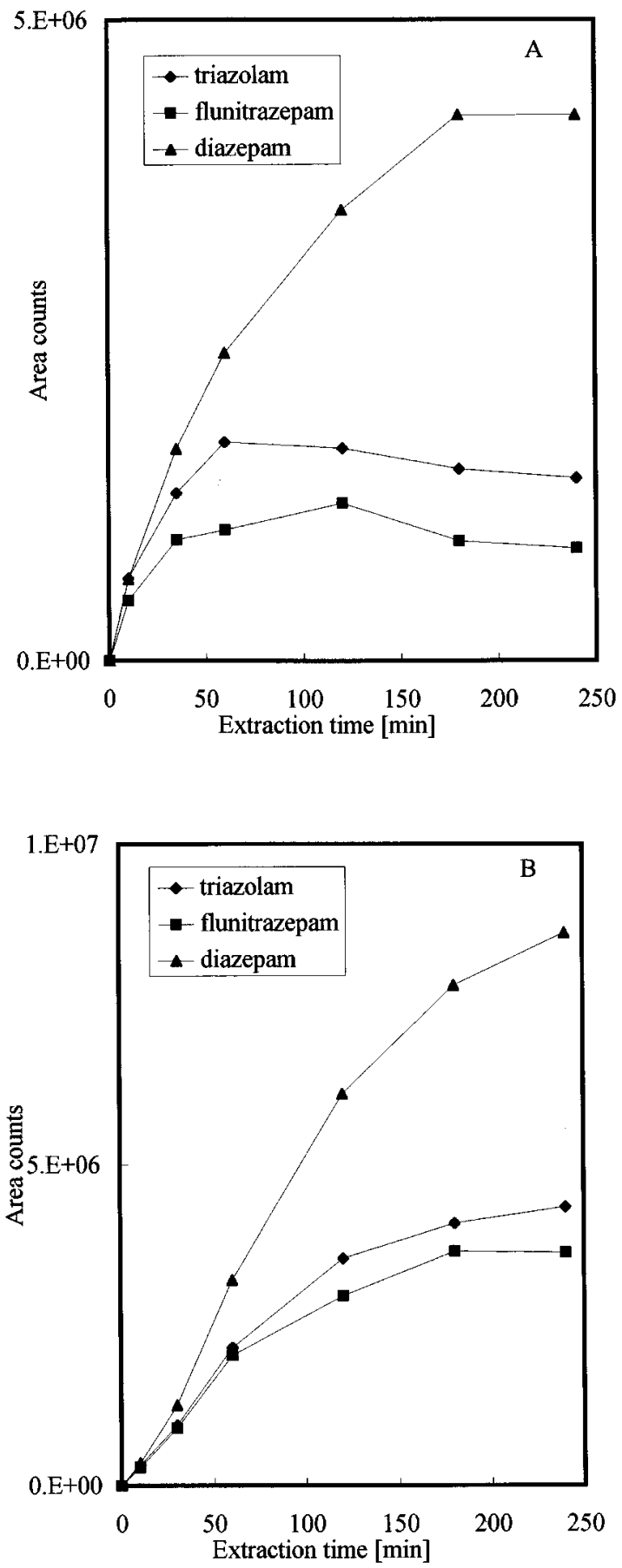

Figure 2. Extraction time profiles with different SPME fiber coatings. A) CW/TEP, B) sol-gel $C_{11}$ PDMS. Concentration of each analyte is $0.1 \mu \mathrm{g} / \mathrm{mL}$. Extraction temperature; $60^{\circ} \mathrm{C}$, desorption time; $30 \mathrm{~min}$, desorption solvent; mobile phase (acetonitrile/ $5 \mathrm{mM}$ phosphate buffer $=40 / 60$ ). examined as desorption solvent, and then the carryover using two coatings such as the sol-gel $\mathrm{C}_{11}$ and the CW/TEP was measured. The results are as follows: the carryover of triazolam, flunitrazepam and diazepam was $6.3,8.3$ and $11.9 \%$ with the CW/TEP and 10.6, 6.5, and $10.1 \%$ with the sol-gel $\mathrm{C}_{11}$, respectively. The results clearly showed that the preheating desorption solvent induced the reduction of the carryover amount and enhanced the total efficiency in SPME process. Therefore the mobile phase preheated at $60{ }^{\circ} \mathrm{C}$ was used as desorption solvent in further evaluation.

In order to see the actual performance of this SPME/micro-LC system for the analysis of benzodiazepines in urine, the CW/TEP coating was used by its better performance in extraction speed with reasonable extraction efficiency, although the alternative sol-gel $\mathrm{C}_{11}$ coating gave better extraction efficiency than that coating. As the first experiment three drugs as the representative benzodiazepines such as triazolam, flunitrazepam, and diazepam were spiked in a controlled-urine as the $0.1 \mu \mathrm{g} / \mathrm{mL}$ concentration and the sample was then diluted 10 -fold by the phosphate buffer $(\mathrm{pH}=6.8)$ for the actual SPME/micro-LC analysis. The analytical results are summarized in figure 3. Figure $3 \mathrm{~A}$ shows the chromatogram by SPME/micro-LC analysis for the urine sample and figure $3 \mathrm{~B}$ was obtained from the blank urine by the SPME/micro-LC. Figure 3C shows the chromatogram for the standard drugs sample in $2 \mu \mathrm{g} / \mathrm{mL}$ concentration by using direct sample injection into micro-LC without SPME process. The results clearly indicate that SPME/micro-LC is very effective for this type of analysis. As the second

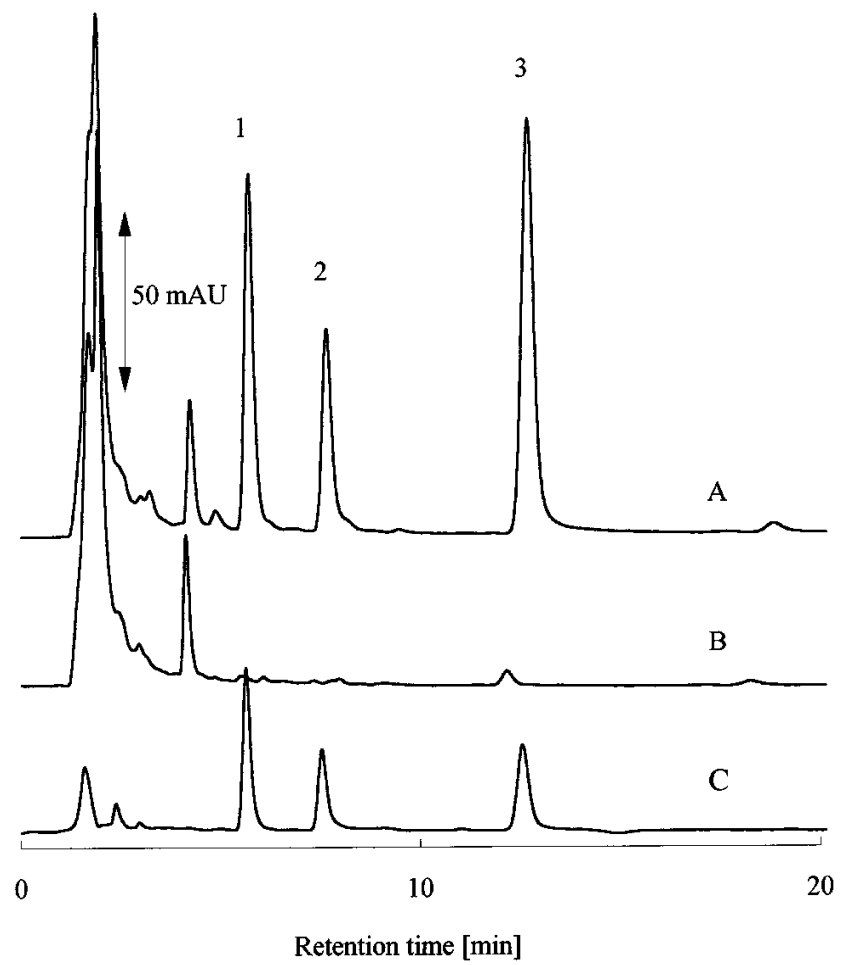

Figure 3. Chromatograms of controlled-urine sample spiked by standard drugs. A) SPME/micro-LC $(0.1 \mu \mathrm{g} / \mathrm{mL})$, B) blank SPME/micro-LC, C) direct LC $(2 \mu \mathrm{g} / \mathrm{mL})$ without SPME process for the standard drugs sample. Column: CAPCELL PAK ODS $1.0 \mathrm{~mm}$ i. d. $\times 150 \mathrm{~mm}$. 1; triazolam, 2; flunitrazepam, 3; diaze- 
experiment to confirm the high performance of the SPME/micro-LC interfacing, the actual patient's urine was probed. The urine was diluted by 10 -fold without any sample pretreatments by the buffer solution and the obtained analytical results are demonstrated in figure 4. Figure 4A shows the chromatogram for the patient's urine by the SPME/micro-LC analysis. In comparison to the chromatogram for standard drugs sample (each $2 \mu \mathrm{g} / \mathrm{mL}$ concentration) without SPME process shown in figure 4B, the peak indicated by the arrow in figure $4 \mathrm{~A}$ can be easily identified as triazolam. Therefore one can conclude by this result that triazolam induced its clinical situation by some reasons such as self-intention or criminal intention. Although the identification in this case is only based on the retention time matching to the standard sample, the SPME/micro-LC offers much better feasibility to couple to other spectroscopic identification method such as mass spectrometry (MS) because low mobile phase flow rate can be realized much easier interfacing between LC and MS.

\section{Conclusions}

In this study it has been clearly suggested that small internal diameter LC column such as $1.0 \mathrm{~mm}$ i.d. can be useful for the analysis of benzodiazepines in human urine sample by coupling to the solventless sample preparation technique, SPME, and reduce the total consumption of organic solvent in analytical procedure. Three fiber coatings for SPME process such as PA, CW/TEP and sol-gel $\mathrm{C}_{11}$ PDMS were compared in their extraction performance such as extraction efficiency and extraction time required. Although the sol-gel $\mathrm{C}_{11}$ PDMS was experimentally found to offer the highest extraction efficiency for benzodiazepines in aqueous media, the CW/TEP fiber coating can give more rapid extraction equilibrium. Therefore the latter is much suitable for the actual sample analysis because analytical speed is the first priority in clinical emergency room analysis and many toxicological analytical situation. By using the CW/TEP coating as the SPME fiber and $1.0 \mathrm{~mm}$ i.d. column for the separation rapid and solventless analytical procedure is realized for the toxicological and forensic drug analysis. The method proposed requires the analysis time less than 2 hours with consuming organic solvents less than $1.5 \mathrm{~mL}$.

\section{References}

1. Taylor, R. B.; Reid, R. G.; Behrens, R. H.; Kanfer, I. J. Pharm. Biomed. Anal. 1992, 10, 867-887.

2. Arthur, C. L.; Pawliszyn, J. Anal. Chem. 1990, 62, 2145- 2148.

3. Chai, M.; Pawliszyn, J. Environ. Sci. Technol. 1995, 29, 693701.

4. Gorecki, T.; Mindrup, R.; Pawliszyn, J. Analyst 1996, 121, 1381-1386.

5. Vaes, W. H. J.; Ramos, E. U.; Verhaar, H. J. M.; Seinen, W.; Hermens, J. L. M. Anal. Chem. 1996, 68, 4463-4467.

6. Valor, I.; Molto, J. C.; Apraiz, D.; Font, G. J. Chromatogr. A 1997, 767, 195-203.

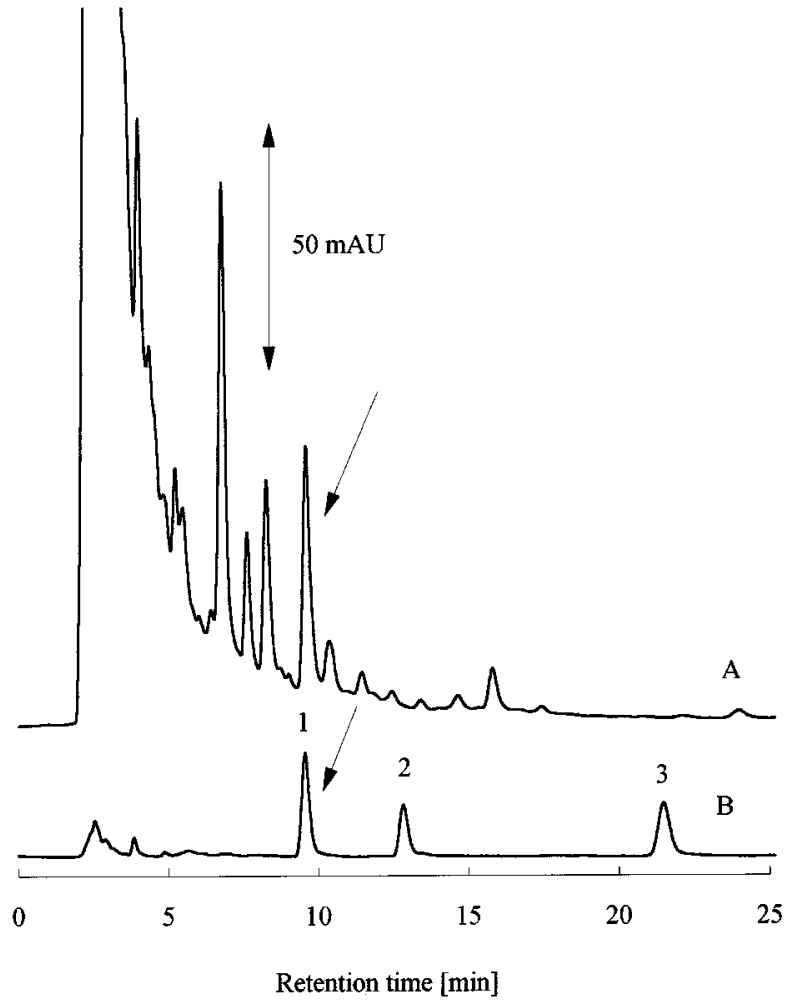

Figure 4. Chromatograms obtained for actual patient's urine sample by SPME/micro-LC and direct LC analysis. A) SPME/micro-LC for the patient's urine, B) direct LC analysis $(2 \mu \mathrm{g} / \mathrm{mL})$ without SPME process for the standard drugs sample. Column: CAPCELL PAK ODS $1.0 \mathrm{~mm}$ i. d. $\times 250 \mathrm{~mm}$. 1; triazolam, 2; flunitrazepam, 3; diazepam.

7. Martos, P. A.; Pawliszyn, J. Anal. Chem. 1997, 69, 206-215.

8. Jinno, K.; Muramatu, T.; Saito, Y.; Kiso, Y.; Magdic, S.; Pawliszyn, J. J. Chromato gr. A 1996, 754, 137-144.

9. Liao, J. L.; Zeng, C. M.; Hjerten, S.; Pawliszyn, J. J. Microcolumn Separations 1996, 8, 1-4.

10. Eisert, R.; Pawliszyn, J. Anal. Chem. 1997, 69, 3140-3147.

11. Nguyen, A. L.; Luong, J. H. T. Anal. Chem. 1997, 69, 17261731.

12. Jinno, K.; Han, Y.; Sawada, H.; Taniguchi, M. Chromatographia 1997, 46, 309-314.

13. Jinno, K.; Taniguchi, M.; Hayashida, M. J. Pharm. Biomed. Anal., in press.

14. Cataby, A.; Taniguchi, M.; Jinno, K.; Pesek, J. J.; Williamsen, E. J. Chromatgr. Sci. 1998, 36, 111-118.

15. Chong, S. L.; Wang, D.; Hayes, J. D.; Wilhite, B. W.; Malik, A. Anal. Chem. 1997, 69, 3889-3898.

16. Boyd-Boland, A. A.; Pawliszyn, J. Anal. Chem. 1996, 68, 1521-1529.

17. Pawliszyn, J. Solid Phase Micro Extraction Theory and Practice, Wiley and Sons: New York, 1997.

18. Daimon, H.; Pawliszyn, J. Anal. Commun. 1997, 34, 365-369. 